
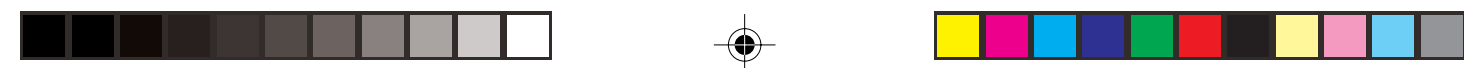

\title{
ESTUDIO COMPARATIVO DE LOS PATRONES DE RIQUEZA ALTITUDINAL DE ESPECIES EN MASTOFAUNAS DE ÁREAS MONTAÑOSAS MEXICANAS
}

\author{
DAVID MONTEAGUDO SABATÉ Y LIVIA LEÓN PANIAGUA
}

Museo de Zoología “Alfonso L. Herrera”, Facultad de Ciencias, Universidad Nacional

Autónoma de México. Apartado Postal 70-399, México D.F., 04510.

Resumen: Se estableció un estudio comparativo de la diversidad mastofaunística de cinco unidades montañosas mexicanas (Sierra de Juárez, Reserva de la Biosfera El Cielo, Sierra Gorda, Sierra de Atoyac de Álvarez, y Reserva de la Biosfera La Sepultura), a partir de transectos altitudinales muestreados en cada una de ellas. Murciélagos y roedores fueron los órdenes más ricos en especies en todos los casos, excepto en la Reserva La Sepultura, donde destacó la gran abundancia de carnívoros en todos los hábitats. Se observó la coincidencia de los patrones generales de riqueza para los mamíferos en las distintas sierras, con una disminución del número de especies con la altitud, tendencia más marcada en los grupos voladores. Asimismo se analizó la similitud entre las faunas de mamíferos de las distintas unidades, y se observó a un primer nivel (unidades fisiográficas) un mayor parecido entre las faunas con componentes pertenecientes a la vertiente atlántica y Chiapas, que se agruparon entre ellas de acuerdo a relaciones de cercanía geográfica, siendo la más alejada de las otras la mastofauna de la Sierra de Atoyac de Álvarez, de la vertiente pacífica, que fue la más pobre en especies. En otro nivel de estudio, se analizaron los agrupamientos entre todos los tipos de vegetación, intervalos altitudinales, en el conjunto de áreas montañosas. Se obtuvo una situación más compleja, donde la importancia del factor altitudinal destaca sobre la unidad fisiográfica concreta, y se observa un división entre las vertientes pacífica y atlántica.

Palabras clave: Mamíferos, México, biodiversidad, similitud faunística, patrones altitudinales.

\section{INTRODUCCIÓN}

México es un país megadiverso, tercero del mundo por su diversidad biológica (Mittermeier, 1988). Esa elevada diversidad se debe, entre otras características, a su ubicación geográfica con la zona de contacto de las biotas neártica y neotropical, la orografía de su territorio y la extraordinaria heterogeneidad del medio físico. Los sistemas montañosos convergen hacia el sur y sureste de México. 
En este contexto, la fauna de mamíferos del país es una de las más diversas del mundo, con 529 especies (Ceballos et al., 2002), riqueza sólo superada por la mastofauna de Brasil e Indonesia. A este gran número de especies contribuye de manera importante la existencia de muchas especies endémicas, especialmente roedores y quirópteros de reducidas áreas de ditribución (Ceballos y Rodríguez, 1993).

En términos generales, la diversidad de especies incrementa de norte a sur, con un valor máximo en la confluencia de la Sierra Madre del Sur, el Eje Neovolcánico y la Sierra Madre Oriental (Espinosa et al., 2000). Sin embargo, el conocimiento de la fauna de regiones montañosas es muy incipiente, lo que limita la descripción de patrones generales. Ello es particularmente importante desde el punto de vista que estas áreas montañosas permiten la existencia de muchos endemismos (Ceballos y Rodríguez, 1993). Esperamos que los patrones sean similares a los reconocidos con otros grupos zoológicos en función que la evolución de éstos debe reflejar la del área en que se encuentran (Nelson y Platnick, 1981), si bien desde un punto de vista ecológico los mamíferos podrían representar un grupo que por sus características fisiológicas (homeotermia, tamaño corporal) difirieran algo en sus patrones de distribución. Por ésta y otras características se suelen estudiar independientemente las faunas de mamíferos voladores y no voladores, que suelen presentar patrones distintos (Arita, 1993; Fa y Morales, 1993; León y Romo, 1993).

El presente trabajo lleva a cabo por primera vez la comparación de áreas montañosas mexicanas atendiendo a la distribución de las mastofaunas correspondientes en transectos sobre las mismas. Es un tema de estudio importante para conocer el comportamiento de la diversidad biológica en estas áreas, la correspondencia o no de los patrones individualizados de cada una de ellas con otros taxones en áreas similares en latitudes tropicales, donde se observa una mayor diversidad en altitudes intermedias (Haber, 1978; Llorente, 1983; Monteagudo et al., 2001; Whittaker y Niering, 1975), y gracias al estudio comparativo entre ellas conocer la distribución de estos patrones de diversidad en un territorio mayor, según la agrupación de los distintos inventarios. En efecto, características generales de humedad y otras suelen identificar un primer nivel de separación de las biotas mexicanas entre los inventarios provenientes de la cuenca del Golfo de México y de la Cuenca el Pacífico pero ello se ve modificado en ciertas altitudes, donde la influencia de factores históricos (Halffter, 1987; Toledo, 1988), es más importante en la definición de las mismas biotas. Todo ello conforma una situación compleja, a cuya descripción colaboramos con este trabajo.

La descripción de la diversidad beta (Magurran, 1988), en forma de similitud entre la combinación de intervalos altitudinales pertenecientes a las distintas unidades geográficas, permite observar el parecido entre las distintas áreas estudiadas e inferir explicaciones acerca del patrón identificado y la importancia del "factor altitudinal" en la definición de las faunas de mamíferos estudiadas. 


\section{MÉTODOS}

Las áreas de estudio corresponden a distintas unidades montañosas dentro del territorio de la República Mexicana, que se seleccionaron a partir de distintos trabajos, teniendo en cuenta la existencia de un muestreo suficiente en las distintas altitudes de dichas áreas, y la posibilidad de comparar los datos procedentes de los mismos.

Gran parte de los datos son procedentes de trabajo de campo del grupo de Mastozoología del Museo de Zoología de la Facultad de Ciencias (MZFC) de la Universidad Nacional Autónoma de México (UNAM) y, en todo caso, del trabajo de investigadores mexicanos en las áreas, con los cuales se mantiene una constante de trabajo y comunicación, con la compartición de métodos y resultados. Todo ello da una gran confianza a los datos que se comparan en el presente estudio. Para establecer un estudio comparativo como el presente son necesarias las siguientes características comunes a los datos a comparar:

- Un mínimo esfuerzo de muestra y, lógicamente, de resultados en la obtención de los datos. Se tomaron siempre inventarios, o combinaciones de los mismos, que se consideraron bajo esta óptica. En este sentido se decidió trabajar con tipos de vegetación, que se asociaron a intervalos altitudinales (Cuadro 1) no necesariamente coincidentes en las distintas unidades geográficas, para poder incorporar la mayor cantidad de información. En ocasiones estos tipos de vegetación habían sido muestreados en varias localidades. En todo caso, corresponde con los datos más confiables existentes en la literatura reciente con respecto a las mastofaunas de las zonas estudiadas.

- Una combinación similar de métodos en la obtención de los datos. En los muestreos se combinaron siempre el método de trampas y redes aéreas (estas últimas para grupos voladores), el registro de observaciones visuales y de rastros diversos, y la obtención de datos procedentes de la literatura especializada. Ello es porque todos los trabajos previos estaban destinados a la obtención de listas de mamíferos, y su descripción en altitud, lo más completas posible de las áreas montañosas correspondientes.

- Gran confiabilidad en la identificación de los taxones descritos, muchos de ellos identificados por el personal del MZFC u otras instituciones dentro de la UNAM. Para unificar las listas se contrastaron con la "Lista Taxonómica de los Mamíferos Terrestres de México" (Ramírez-Pulido et al., 1996), con respecto a la cual se hicieron las modificaciones necesarias, y que se tomó como la referencia taxonómica para el presente estudio.

Se descartó trabajar con otros datos cuando no correspondían a estas características; por ejemplo en casos de listas puntuales no asimilables un gradiente altitudinal, listas correspondientes a territorios solo definidos políticamente (faunas de Estados o municipios) o claramente con un nivel más bajo de completitud (solo para taxones particulares o informes de trabajo de campo). Se descartó introducir los datos procedentes de la Sierra de Taxco (León y Romo, 1993) aunque el trabajo de campo fue similar por no ser comparables al tratarse de una fauna extraordinariamente 

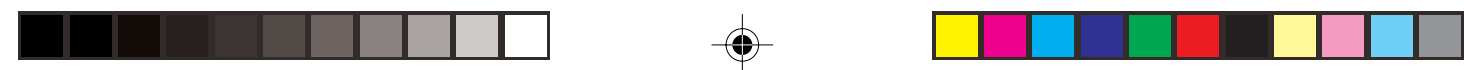

Diciembre 2002 Monteagudo, D. y L. León - Patrones altitudinales...

63

Cuadro 1. Descripción de las unidades estudiadas. Cada unidad de comparación es un tipo de vegetación, o combinación de tipos, al cual se hará corresponder un intervalo altitudinal. Los acrónimos correspondientes a los tipos de vegetación son: SAP, selva alta perennifolia;

BMM, bosque mesófilo de montaña; BPE, bosque de pino-encino; BTS, bosque tropical subcaducifolio; MX, matorral xerófilo; BE, bosque de encino; BJ, bosque de SB, Juniperus; $\mathrm{BP}$, bosque de pino; BA, bosque de Abies y SB, selva baja.

\begin{tabular}{|c|c|c|c|c|}
\hline Acrónimo & Unidad & $\begin{array}{l}\text { Tipo de } \\
\text { Vegetación }\end{array}$ & $\begin{array}{l}\text { Altitud } \\
\text { (msnm) }\end{array}$ & $\begin{array}{l}\text { Total de } \\
\text { especies }\end{array}$ \\
\hline SJ1 & S. Juárez & SAP & $100-150$ & 47 \\
\hline SJ2 & S. Juárez & SAP BMM & $650-900$ & 29 \\
\hline SJ3 & S. Juárez & BMM & $1,700-1,900$ & 33 \\
\hline SJ4 & S. Juárez & BMM BPE & 2,300 & 9 \\
\hline EC1 & El Cielo & BTS & $200-800$ & 65 \\
\hline EC2 & El Cielo & BMM & $800-1,400$ & 50 \\
\hline EC3 & El Cielo & BPE & $1,400-1,800$ & 23 \\
\hline EC4 & El Cielo & MX & $1,300-1,600$ & 28 \\
\hline SQ1 & S. Gorda & BT & $800-1,000$ & 41 \\
\hline SQ2 & S. Gorda & MX & $1,330-1,840$ & 36 \\
\hline SQ3 & S. Gorda & $\mathrm{BE}$ & 1,440 & 32 \\
\hline SQ4 & S. Gorda & BJ & 2,190 & 11 \\
\hline SQ5 & S. Gorda & $\mathrm{BP}$ & 1,650 & 30 \\
\hline AA1 & S. Atoyac & BTS & $680-820$ & 25 \\
\hline AA2 & S. Atoyac & $\begin{array}{l}\text { BTS BMM } \\
\text { (ecotono) }\end{array}$ & 1,200 & 13 \\
\hline AA3 & S. Atoyac & BMM & $1,400-2,000$ & 27 \\
\hline AA4 & S. Atoyac & BPE BMM & $2,200-2,500$ & 29 \\
\hline AA5 & S. Atoyac & BA & 3,100 & 7 \\
\hline LS1 & La Sepultura & SB & $400-500$ & 57 \\
\hline LS2 & La Sepultura & BMM & $927-1,380$ & 58 \\
\hline LS3 & La Sepultura & $\mathrm{BPE}$ & $835-1,700$ & 54 \\
\hline
\end{tabular}


empobrecida donde no se reflejan patrones generales de distribución altitudinal. Finalmente se determinó que el estudio comparativo podía definirse entre las siguientes unidades montañosas: Sierra de Atoyac de Álvarez (Guerrero), Sierra Gorda (Querétaro), Sierra de Juárez (Oaxaca), Reserva de la Biosfera "El Cielo" (Tamaulipas) y Reserva de la Biosfera "La Sepultura" (Chiapas). Una descripción más detallada de las unidades de estudio, su ubicación, las localidades y tipos de vegetación muestreados y otras se hallan en el siguiente apartado.

Se construyó inicialmente la matriz de datos correspondiente, que muestra en las distintas columnas la identidad del taxón, a nivel específico, y en forma de datos de presencia (1) o ausencia (0) su situación en las distintas unidades geográficas operativas, OGUs (Murguía y Rojas, 2001). Estas OGUs se corresponden con los tipos de vegetación descritos en cada una de las sierras, atendiendo en todos los casos a la clasificación de Rzedowski (1978) y se asigna un intervalo altitudinal a cada una de ellas. En algunos casos es más clara esta correspondencia, y en otros existe un importante grado de sobrelapamiento entre los distintos tipos de vegetación (p. ej. en la Sierra de Querétaro), complicándose algo esta correspondencia, debido a que se muestreó en las dos vertientes de la sierra.

Se tomó la riqueza específica (observada) como medida de la diversidad a para cada OGU, y se calculó la diversidad a promedio para cada cadena montañosa. Se compararon los resultados obtenidos. Asimismo se obtuvieron los datos de diversidad gamma (total de especies de mamíferos) para cada sierra.

Se analizaron los resultados obtenidos en las distintas altitudes de las unidades muestreadas, los cuales se representaron gráficamente en una figura mostrando las altitudes promedio (eje de ordenadas) y la riqueza específica (eje de abscisas) para todas las unidades, para inferir la existencia de un patrón general de la diversidad de la mastofauna con la elevación. Para ello se obtuvieron dos gráficas, una para el total de mamíferos y otra para los mamíferos voladores.

Posteriormente se hizo un análisis de la similitud mastofaunística entre los inventarios procedentes de las distintas situaciones. Para ello se utilizó el índice de Sorensen,

$$
\mathrm{QS}=2 \mathrm{~s} /\left(\mathrm{n}_{1}+\mathrm{n}_{2}\right)
$$

donde "s" es el número de especies compartidas por cada par de inventarios y " $n_{1 "} \mathrm{y}$ " $n_{2}$ " es el número de especies de cada uno de los inventarios individuales a comparar.

Éste es uno de los índices más habituales en este tipo de estudios (Pielou, 1975; Magurran, 1988) y al dar mayor peso a las especies compartidas contribuye a la definición de los agrupamientos. Se aplicó a cada par de inventarios posible para establecer la similitud entre los mismos. Los resultados se representaron en una matriz de similitudes para las distintas OGUs (Cuadro 2 y 3).

A partir de la matriz de similitud se procedió al análisis de agrupaciones, con la construcción del fenograma correspondiente, utilizando la técnica del ligamiento promedio no ponderado (UPGMA). Este modo de agrupación deforma menos las relaciones originales entre las distintas unidades en comparación en la construcción 
del dendrograma (Crisci y López-Armengol, 1983). Para ello se utilizó el paquete informático Ntsys versión 1.80 (Applied Biostatistics Inc., 1993, 1994).

\section{Área de estudio}

Sierra de Juárez (SJ), Oaxaca. Ubicada entre los $17^{\circ} 29^{\prime}$ y los $17^{\circ} 54^{\prime}$ latitud norte y los $97^{\circ} 44^{\prime}$ longitud oeste, se encuentra en la región septentrional del estado, en la provincia de las Serranías Meridionales en la región Mesoamericana de Montaña (Fig. 1). Se trata de un área de topografía muy accidentada con pocas interrupciones de terrenos planos o pendientes suaves. Al menos una parte podría considerarse como una prolongación de la Sierra Madre Oriental, que queda interceptada por el Eje Neovolcánico. Entre sus cumbres destaca el cerro Zempoaltépetl (3,400 msnm). En la mayor parte del sistema montañoso predominan altitudes superiores a los 1,000. Los datos proceden del trabajo de campo realizado por el MZFC durante los años 1996 a 1997 (Peña-Hurtado, en prep.), en que se identificaron 69 especies de mamíferos.

Reserva de la Biosfera "El Cielo" (EC), Tamaulipas. Se localiza al suroeste del estado, entre $22^{\circ} 55^{\prime}$ y $23^{\circ} 25^{\prime}$ latitud norte y $99^{\circ} 05^{\prime}$ y $99^{\circ} 26^{\prime}$ longitud oeste. Abarca parte de la Sierra de Cucharas o de Guatemala, macizo divergente de la Sierra Madre Oriental y una serie de pequeñas sierras (Sánchez-Ramos, 1992). Vargas-

Cuadro 2. Valores del Índice de Sorensen ( triángulo superior) y el número de especies de mamíferos compartidas (triángulo inferior), para cada par de inventarios, donde OGU corresponde al total de la Sierra.

\begin{tabular}{llllll}
\hline & SJ & EC & SQ & AA & LS \\
\hline SJ & $\mathrm{x}$ & 34 & 26 & 19 & 40 \\
EC & 0.42 & $\mathrm{x}$ & 54 & 26 & 44 \\
SQ & 0.35 & 0.62 & $\mathrm{x}$ & 22 & 32 \\
AA & 0.33 & 0.37 & 0.35 & $\mathrm{x}$ & 23 \\
LS & 0.56 & 0.52 & 0.41 & 0.38 & $\mathrm{x}$ \\
& & & & & \\
\hline
\end{tabular}




\begin{tabular}{|c|}
\hline 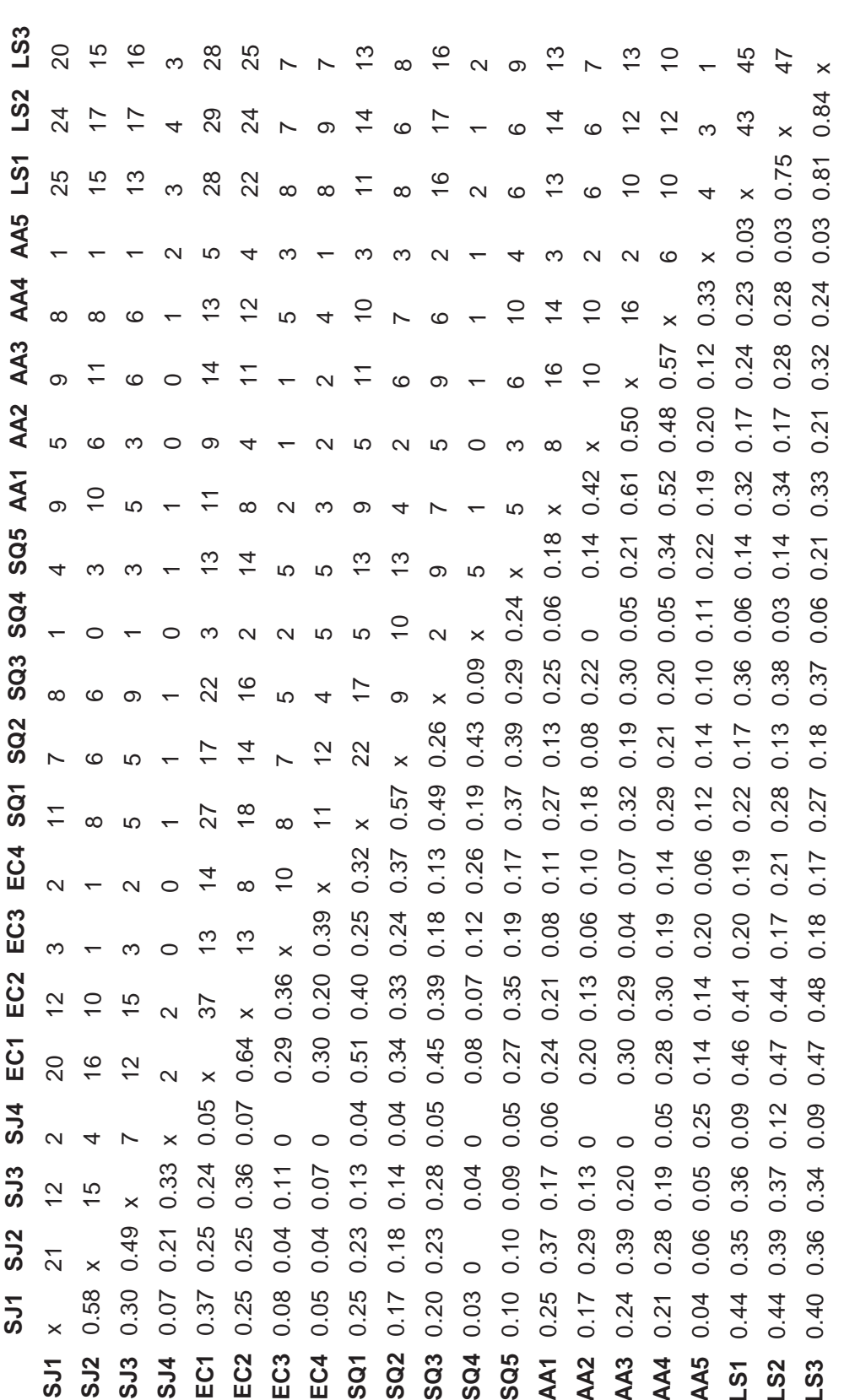 \\
\hline
\end{tabular}


Contreras y Hernández-Huerta (2001) publicaron la lista de la mastofauna de la reserva, obteniéndose un total de 96 especies registradas a partir de datos de campo (varios métodos directos e indirectos) entre 1990 y 1996, y consulta de distintas bases de datos y colecciones. A efectos del presente trabajo se agruparon los registros con base en cuatro tipos de vegetación: bosque tropical subcaducifolio (200-800 m de altitud), bosque mesófilo de montaña (800-1,400 m), bosque de pino y encino (1,400 a más de 1,800 m), con predominancia de unas u otras especies de árboles según altitud y matorral xerófilo (entre 1,300 y 1,600 m).

Sierra Gorda (SQ), Querétaro. Se encuentra situada al noreste del Estado de Querétaro. Se muestrearon localidades entre los $22^{\circ} 00^{\prime}$ y $21^{\circ} 18^{\prime}$ latitud norte y los 9906’ y 9955', pertenecientes a la vertiente atlántica y pacífica, confluencia de cuencas donde podemos ubicar la unidad. El estudio de campo sobre la mastofauna del área fue desarrollado por el MZFC entre los años 1982 a 1990, derivándose del mismo varias publicaciones (León, 1986; León et al., 1990; Navarro y León, 1995; Romo, 1993) de las que proceden los datos utilizados en este trabajo. Se obtuvo la distribución de 69 especies registradas para el área.

Sierra de Atoyac de Álvarez (AA), Guerrero. Se localiza entre los $17^{\circ} 15^{\prime}$ y $17^{\circ} 45^{\prime}$ latitud norte y los $100^{\circ} 10^{\prime}$ y $100^{\circ} 20^{\prime}$ longitud oeste, perteneciente al Sistema Orográfico Meridional del estado de Guerrero (Figueroa de Contín, 1980), en la Sierra Madre del Sur, unidad de la cual incluye las mayores altitudes como el Cerro Teotepec, 3,100 msnm. La topografía es muy accidentada, presentando barrancas y cañadas de alta pendiente. Se obtuvieron los datos de recolección de mamíferos en 11 localidades, pertenecientes a los municipios de Atoyac de Álvarez, Heliodoro Castillo y San Miguel Totolapan, que representan un transecto altitudinal de 2,400 m. Aqui se presentan los datos de ocho localidades que ofrecen un muestreo suficiente, agrupadas atendiendo a los tipos de vegetación descritos: bosque tropical subcaducifolio (680 $820 \mathrm{~m}$ ), bosque tropical subcaducifolio-bosque mesófilo de montaña(1,200 m), bosque mesófilo de montaña $(1,400-2,000 \mathrm{~m})$ y bosque de pino encino-bosque mesófilo de montaña $(2,200$ - 2,500 m). El muestreo se llevó a cabo entre los años 1985 y 1987. Los datos utilizados, inéditos, proceden del trabajo del MZFC, y representan un total de 46 especies. Hasta la fecha solamente se ha publicado la lista de los roedores del área (Juárez, 1993).

Reserva de la Biosfera "La Sepultura" (LS), Chiapas. Situada en la porción noroeste de la Sierra Madre de Chiapas, en la región sur del estado, al norte de la llanura costera del Pacífico. La reserva está al sureste del estado, entre los $16^{\circ} 00^{\prime}$ y $16^{\circ} 29^{\prime}$ latitud norte y $93^{\circ} 24^{\prime}$ y $94^{\circ} 87^{\prime}$ longitud oeste, con una superficie de 167,309 hectáreas entre los municipios de Arriaga, Cintalapa, Jiquipilas, Tonalá, Villa Corzo y Villaflores (SEMARNAP, 1999). Se trata de un ára de compleja orografía, y los datos presentados comprenden un rango altitudinal entre los 200 y 1,600 m, en quince localidades. Los datos utilizados proceden de Riechers y Vázquez (en preparación), quienes realizaron el inventario de mamífero de la reserva a partir de revisiones bibliográficas, bases de datos de colecciones y trabajo de campo (marzo de 1998 a 

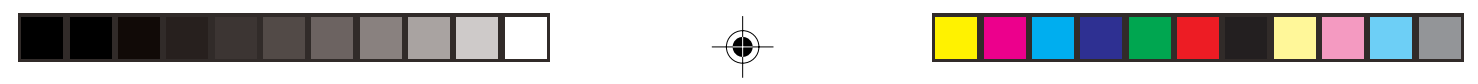

junio de 1999) que comprendió observaciones directas e indirectas y distintos métodos de recolecta apropiados a los distintos grupos muestreados (redes, trampas), obteniéndose una riqueza de 75 especies de mamíferos para el área. Asimilamos los mismos datos a tres tipos de vegetación: selva alta perennifolia (900 - 1,600 m), bosque de pino y encino (835 a 1,700 m) y bosque mesófilo de montaña (927 a 1,500 m).

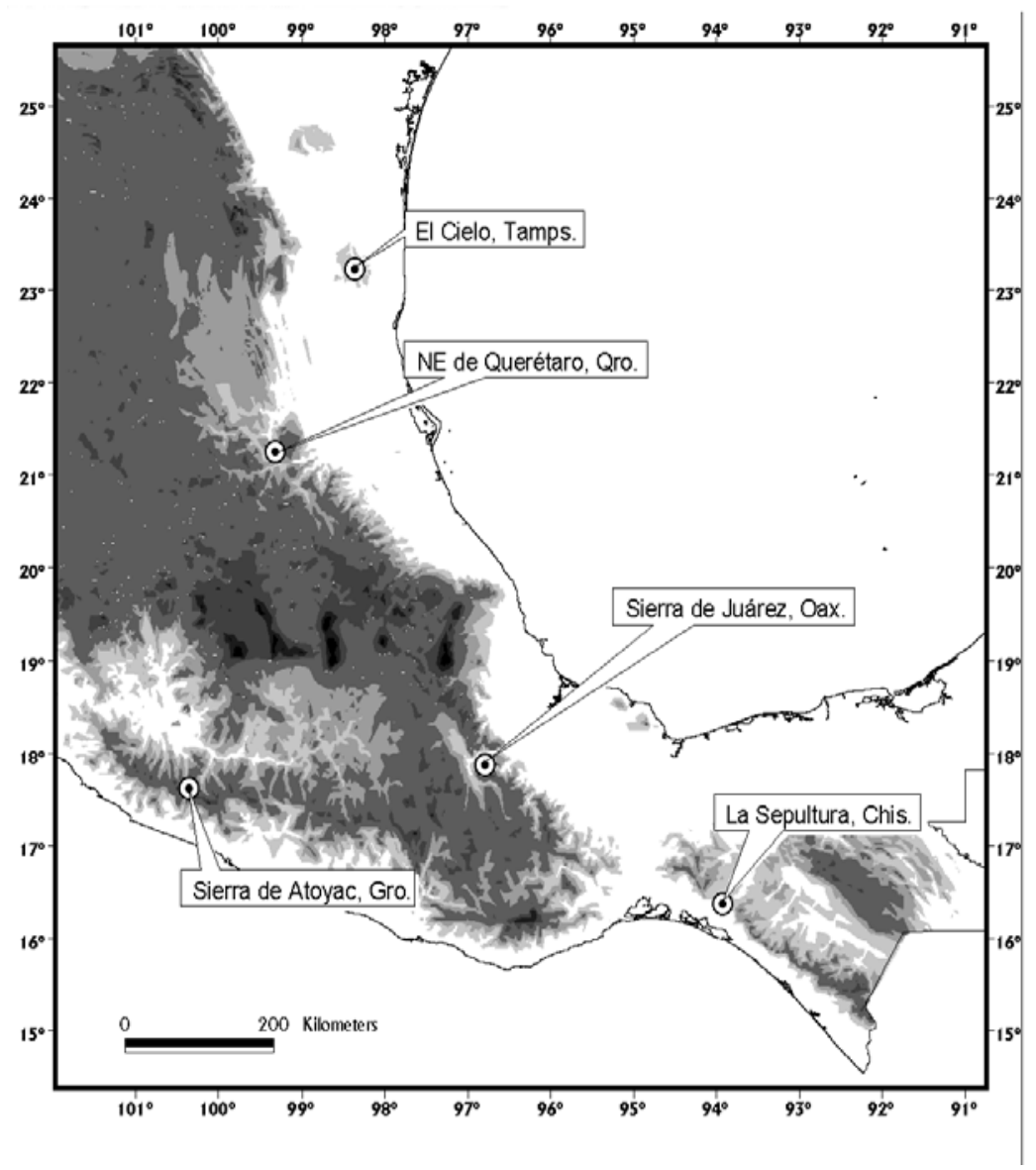

Figura 1. Ubicación geográfica de las áreas del estudio comparativo. 


\section{RESULTADOS Y DISCUSIÓN}

La riqueza para el total de Mamíferos y murciélagos disminuyó con la altitud, de manera casi lineal en algunos casos ("El Cielo” y Sierra de Juárez), y bajo un patrón más complejo en otros (Fig. 2). En el caso de la Reserva de la Biosfera La Sepultura La tendencia a la disminución de la diversidad con la elevación fue más marcada tomando en cuenta solamente los murciélagos, característica que también se destaca en otros trabajos (Arita, 1993; Sánchez-Cordero, 2001). Esta tendencia se debe a la mayor capacidad de desplazamiento sobre todo en las altitudes inferiores,hábitos alimentarios y razones fisiológicas.

Con frecuencia se destaca que la diversidad en las áreas tropicales encuentra su punto máximo en elevaciones intermedias, ligeramente variables a lo largo del eje latitudinal. Ello ha sido demostrado para numerosos grupos zoológicos (Aves, Terborgh, 1977; Ithomiinae, Haber, 1978; Dismorphiinae, Llorente, 1983; Papilionoidea en general, Monteagudo et al., 2002), y puesto de manifiesto como patrón más generalizable (Whittaker y Niering, 1975; Janzen et al., 1976). Lo mismo puede inferirse del estudio de Fa y Morales (1993), quienes mencionan el patrón de diversidad de mamíferos en elevación como reflejo del patrón latitudinal de riqueza que muestra un máximo en México sobre los $20^{\circ}$ latitudinales. No observamos esta característica en el presente estudio para comunidades de mamíferos, aún cuando la capacidad endotérmica del grupo parecería indicar incluso que este pico de diversidad pudiera desplazarse a elevaciones algo superiores, de acuerdo al reflejo del patrón latitudinal descrito. Ello coincide con los resultados obtenidos por Navarro (1992) en un transecto altitudinal con aves, otro grupo endotérmico, sobre la sierra de Atoyac de Álvarez.

Las figuras 3 y 4 muestran los dendrogramas identificados a partir del análisis de agrupamientos, para las cinco unidades fisiográficas utilizadas en este trabajo (Fig. 3), y para las distintas unidades de vegetación descritas en todas las unidades en conjunto, sobre las que se asimila un determinado intervalo altitudinal (Fig. 4). EC y SQ presentaron una importante similitud (QS = 0.62). En ello influye la cercanía entre las sierras y la continuidad entre la Sierra Madre Oriental y la Sierra de Querétaro, dentro de la misma provincia morfotectónica (Ferrusquía-Villafranca, 1993). A un nivel de similitud algo menor se relacionan la Sierra de Juárez y La Sepultura. La Sierra de Juárez se encuentra en la vertiente atlántica, y si bien la Reserva de la Biosfera La Sepultura se halla en la vertiente pacífica, las características de humedad son similares por razones orográficas principalmente. Chiapas y Oaxaca son los estados más biodiversos de la República (Flores y Gerez, 1994), lo cual también influye en esta relación. Estos dos bloques se agrupan en un nivel de similitud del $42 \%$ en el dendrograma, relacionándose así por la influencia sobre todo de una mayor riqueza y características de humedad. Por último, la mastofauna que presenta menor relación con el resto es la de la sierra de Atoyac de Álvarez, en la vertiente pacífica, con una comunidad más pobre. 


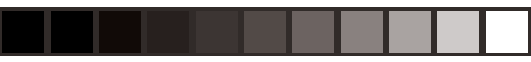

vol. 6

Figura 2. Patrones altitudinales de distribución de riqueza en las unidades geográficas estudiadas. En el eje X se representa la altitud promedio de cada unidad estudiada, en el eje Y el número de especies identificado. Total de mamíferos (arriba) y murciélagos (abajo).

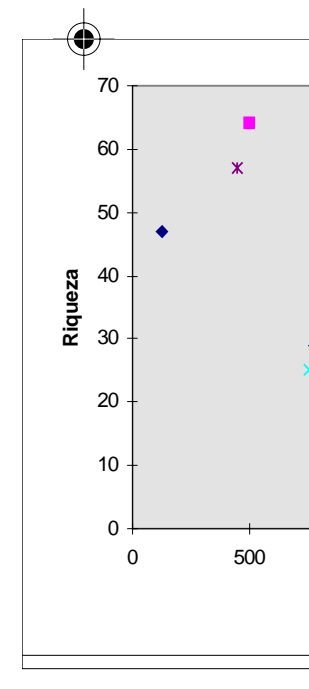


La figura 4 muestra las relaciones entre las distintas unidades de muestreo, cuando éstas corresponden a los distintos tipos de vegetación (intervalos altitudinales) en todas las unidades fisiográficas. Se observa una situación más compleja que la unión por unidades fisiográficas. La fauna más diferenciada corresponde a la unión de los intervalos altitudinales superiores de la Sierra de Juárez y de Atoyac de Ávarez, por encima de los 2,300 msnm. Cabe destacar que la unión de estos inventarios se da sobre todo por el hecho que representan dos faunas muy pobres, y no por la compartición de de especies (presentan un nivel muy bajo de similitud). En general, en estas altitudes superiores destacamos pues la pobreza de especies, y que en la mayoría de los casos se trata de especies comunes. Solo Hodomys alleni se presenta exclusivamente en este intervalo, en la sierra de Atoyac de Álvarez. Pese a esta pobreza general de especies en grandes elevaciones, Peterson et al. (1993) destacan la existencia de gran número de endemismos.

Las otras OGUs se agrupan en dos bloques principales: (1) comprendiendo los inventarios de mayores altitudes de "El Cielo" (formando un subgrupo), pues en esta unidad se aprecia un empobrecimiento faunístico muy marcado a altitudes superiores a 1,400 msnm, y la mayoría de los pertenecientes a la Sierra Gorda (en otro subgrupo), ello viene determinado por la distancia geográfica relativamente pequeña entre las dos unidades fisiográficas; y (2) el resto de inventarios, que se constituyen a su vez en dos subconjuntos: la sierra de Atoyac de Álvarez (excepto la altitud superior) por un lado, y el resto por otro. Estos dos subgrupos podrían representar el agrupamiento de las altitudes inferiores en las áreas de la vertiente pacífica y del Golfo, respectivamente (cabe destacar que el único inventario de Querétaro correspondiente a este bloque es precisamente el bosque de encino, en la vertiente atlántica y con características de mayor humedad que el resto de los muestreados en la sierra, con predominio de la vertiente pacífica). Los inventarios de Chiapas ("La Sepultura”) muestran mayor afinidad con los de la vertiente atlántica, pues sus características climáticas son más parecidas a éstos que al resto de la vertiente pacífica. Dentro de este último bloque, que es el que comprende la mayoría de OGUs, éstas se agrupan con base en las unidades fisiográficas a las que pertenecen, siendo el bloque más compacto el constituido por las de "La Sepultura" $(\mathrm{QS}=0.76)$, porque comprenden un menor transecto altitudinal.

\section{CONCLUSIONES}

La mastofauna de distintas áreas montañosas en México muestra un patrón de comportamiento general de disminución de la diversidad con la elevación, sin que sea apreciable un máximo de riqueza en altitudes intermedias, como describen distintos autores para otros grupos zoológicos, a partir de los datos. Las relaciones entre las distintas unidades fisiográficas siguen, a partir del análisis de similitud para el total de las faunas de cada una de ellas, los patrones generales de separación entre las faunas de la vertiente atlántica y Chiapas y la vertiente pacífica, éstas se muestran más 

generales de las unidades montañosas en conjunto.

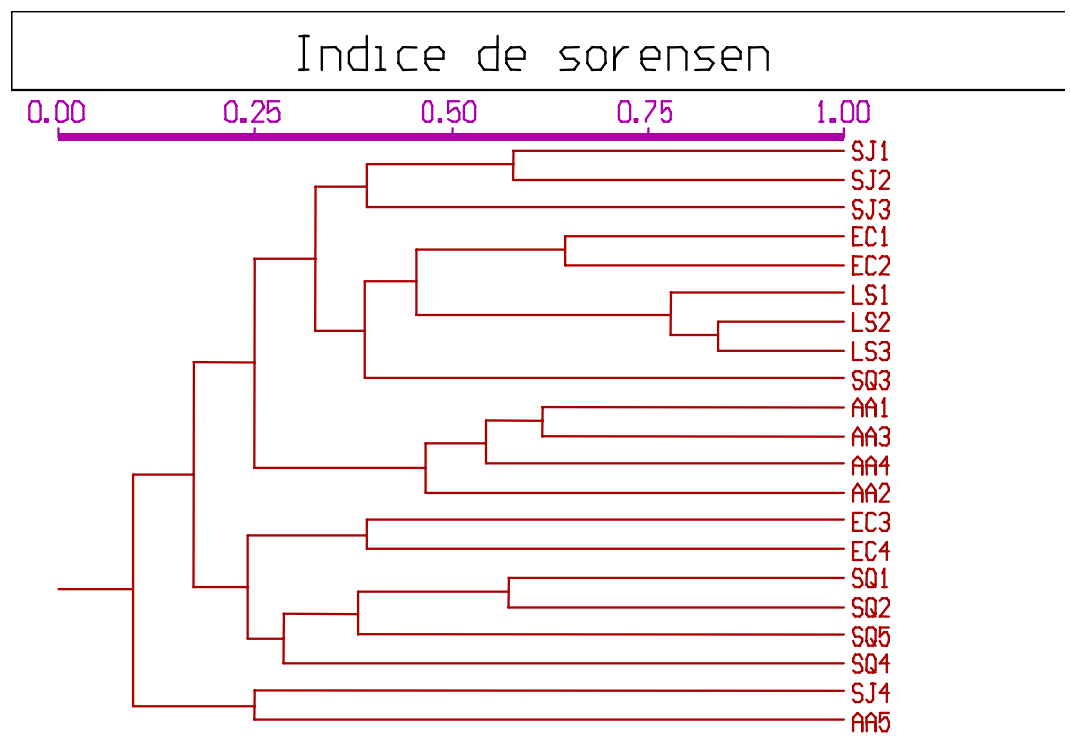

Figura 4. Dendrograma de agrupaciones para la totalidad de unidades de muestreo (tipos de vegetación) de las cinco unidades fisiográficas. 
complejas al analizarlas a nivel altitudinal, donde encontramos un primer nivel de diferencición para las elevaciones superiores, y destacamos la gran influencia del factor de pertenencia de las comunidades a la vertiente atlántica o pacífica para las elaciones faunística, predominante en general frente a la distancia relativa.

\section{AGRADECIMIENTOS}

Al Museo de Zoología de la Universidad Nacional Autónoma de México nos facilitó instalaciones y condiciones de investigación. A L. Peña Hurtado, J.A. Vargas Contreras, A. Hernández Huerta, A. Riechers Pérez y D,. Vázquez, quienes permitieron el uso de los datos procedentes de sus trabajos para la realización del presente. A nuestros compañeros del MZFC A. Navarro Sigüenza y T. Escalante, quienes accedieron amablemente a la revisión del manuscrito original y contribuyeron a mejorarlo con sus acertadas críticas.

\section{LITERATURA CITADA}

Arita, H.T. 1993. Riqueza de especies de la mastofauna de México. Pp. 109-128, en: Avances en el estudio de los mamíferos de México. (Medellín, R.A. y G. Ceballos, eds.) Publicaciones Especiales, Vol. 1, Asociación Mexicana de Mastozoología, A.C., México D.F.

Arita, H.T. y G. Ceballos. 1997. Los mamíferos de México: Distribución y estado de conservación. Revista Mexicana de Mastozoología, 2: 33-71.

Ceballos, G. y P. Rodríguez. 1993. Diversidad y conservación de los mamíferos de México: Patrones de Endemicidad. Pp. 87-108, en: Avances en el Estudio de los Mamíferos de México. (Medellín, R.A. y G. Ceballos, eds.) Publicaciones Especiales, Vol. 1, Asociación Mexicana de Mastozoología, A.C., México D.F.

Ceballos, G.C., J. Arroyo-Cabrales and R. Medellín. 2002. The mammals of Mexico: composition, distribution, and conservation. Occasional Papers, Museum of Texas Tech University, 218:1-27.

Crisci, J.V. y M.F. López-Armengol. 1983. Introducción a la taxonomía y práctica de la taxonomía numérica. Secretaría General de la Organización de los Estados Americanos. Programa Regional de Desarrollo Científico y Tecnológico. Serie de Biología. Monografía 26. Washington D.C.

Espinosa, D., J. J. Morrone, C. Aguilar y J. Llorente. 2000. Regionalización biogeográfica de México: provincias bióticas. Pp. 61-94, en: Biodiversidad, Taxonomía y Biogeografía de Artrópodos de México: Hacia una síntesis de su conocimiento. (J. Llorente, E. González, N. Papavero, eds.). CONABIO, México, D. F.

Fa, J. y L. Morales. 1993. Patterns of mammalian diversity in Mexico. Pp. 319-361, en: Biological diversity of Mexico: origins and distribution. (T.P. Ramamoorthy, R. Bye, A. Lot y J.Fa, eds.) Oxford University Press. Nueva York, E.U.A.

Ferrusquía-Villafranca, I. 1993. Geology of Mexico: A Synopsis. Pp. 3-107, en: Biological diversity of Mexico: origins and distribution. (T.P. Ramamoorthy, R. Bye, A. Lot y J. Fa, eds.) Oxford University Press, Nueva York, E.U.A. 
Figueroa de Contín, E. 1980. Atlas Geográfico e Histórico del Estado de Guerrero. FONAPAS, Gobierno del Estado, Chilpancingo.

Fleming, T. H. 1973. The number of mammal species in several North and Central American forests communities. Ecology, 54: 555-563.

Flores, O. y P. Gerez. 1994. Biodiversidad y conservación en México: vertebrados, vegetación y uso del suelo. Comisión Nacional para el Conocimiento y Uso de la Biodiversidad y Universidad Nacional Autónoma de México. México D.F.

Graham, G. L. 1983. Changes in bat species diversity along an elevational gradient up the Peruvian Andes. Journal of Mammalogy, 64: 559-571.

Haber, W. A. 1978.Evolutionary Ecology of Tropical Mimethic Butterflies (Lepidoptera: Ithomiinae). Tesis doctoral. Universidad de Minessota.

Halffter, G. 1987. Biogeography of the Montane Entomofauna of Mexico and Central America. Annual Review o Entomology, 32: 95-114.

Janzen, D.H. M. Ataroff, M. Fariñas, S. Reyes, N. Rincón, A. Soler, P. Soriano y M. Vera, 1976. Changes in the arthropod community along an elevational transect in the Venezuelan Andes. Biotropica, 8:193-203.

León. L. 1986. Distribución altitudinal de los murciélagos en el Noreste del Estado de Querétaro. Tesis de Licenciatura, Facultad de Ciencias, Universidad Nacional Autónoma de México, México D. F.

León, L. y E. Romo. 1993. Mastofauna de la Sierra de Taxco, Guerrero. Pp. 45-64, en: Avances en el Estudio de los Mamíferos de México. (Medellín, R.A. y G. Ceballos, eds.). 1993. Publicaciones especiales, Vol. 1, Asociación Mexicana de Mastozoología, A.C., México, D.F.

León, L., E. Romo, J.C. Morales, D. Schmidly y D. Navarro. 1990. Noreworthy records of mammals from the state of Querétaro, México. The Southwestern Naturalist, 2: 231-235.

Llorente, J. 1983. Sinopsis sistemática y biogeográfica de los Dismorphiinae de México con especial referencia al género Enantia Hüebner (Lepidoptera: Pieridae). Folia Entomológica Mexicana, 58:1-207.

Luis, A., I. Vargas y J. Llorente. 1991. Lepidopterofauna de Oaxaca I: Distribución y Fenología de los Papilionoidea de la Sierra de Juárez. Publicaciones especiales del Museo de Zoología, 3, Universidad Nacional Autónoma de México, México D.F.

Magurran, A.E., 1988. Ecological diversity and its measurement. Princeton University Press. Princeton, Nueva Jersey, E.U.A.

Mittermeier, R.A., 1988. Primate diversity and the tropical forest: case study from Brazil and Madagascar and the importance of megadiversity countries. Pp. 145-154, en: Biodiversity (E.O. Wilson, ed.). National Academy Press, Washington, D.C., E.U.A.

Monteagudo-Sabaté, D., A. Luis-Martínez, I. Vargas-Fernández y J. Llorente-Bousquets. 2001. Patrones altitudinales de diversidad de mariposas en la Sierra Madre del Sur (México). SHILAP. Revista de Lepidopterología, 29(115):207-237.

Murguía, M. y F. Rojas. 2001. Biogeografía Cuantitativa. Pp. 39-47, en: Introducción a la Biogeografía en Latinoamérica: teorías, conceptos, métodos y aplicaciones. (LlorenteBousquets, J. y J. J. Morrone, eds.). México, D.F.

Navarro, A. 1992. Altitudinal distribution of birds in the Sierra Madre del Sur, Guerrero, México. The Condor, 94:29-39. 
Navarro, D. y L. León. 1995. Community structure of bats along an altitudinal gradient in tropical eastern Mexico. Revista Mexicana de Mastozoología, 1:9-21.

Nelson y Platnick. 1981. Systematics and biogeography, cladistics and vicariance. Columbia University Press, Nueva York, E.U.A.

Peterson, A. T., O. A. Flores-Villela, L. S. León-Paniagua, J. E. Llorente-Bousquets, M. A. Luis-Martínez, A. G. Navarro-Sigüenza, M. G. Torres-Chávez e I. Vargas-Fernández. 1993. Conservation priorities in México: moving up to the World. Biodiversity Letters, 1: 33-38.

Pielou, E.C. 1975. Ecological Diversity. John Wiley \& Sons. Nueva York, E.U.A.

Ramírez-Pulido, J., A. Castro-Campillo, J. Arroyo-Cabrales, F. A. Cervantes. 1996. Lista taxonómica de los mamíferos terrestres de México. Occasional Papers, The Museum, Texas Tech University, 158: 1-62.

Romo, E. 1993. Distribución Altitudinal de los Roedores del Noreste del Estado de Querétaro. Tesis de Licenciatura, Facultad de Ciencias, Universidad Nacional Autónoma de México, México D.F.

Rzedowski, J. 1978. Vegetación de México. Ed. Limusa. México, D.F.

Rzedowski, J. 1998. Diversidad y orígenes de la flora fanerogámica de México. Pp. 129-145, en: Diversidad biológica de México. Orígenes y distribución. (Ramamoorthy, T.P., R. Bye, A. Lot y J. Fa, eds.). Oxford University Press, Nueva York, E.U.A.

Sánchez, O. y G. López. 1988. A theoretical analysis of some indices of similarity as applied to biogeography. Folia Entomológica Mexicana, 75:119-145.

Sánchez-Cordero, V. 2001. Elevation gradients of diversity for rodents and bats in Oaxaca, Mexico. Global Ecology and Biogeography, 10:63-76.

Sánchez-Ramos, G. 1992. La Reserva de la Biosfera "El Cielo”, Antecedentes, Objetivos y Problemática Actual. Biostam, 4(3).

SEMARNAP. 1999. Programa de Manejo Reserva de la Biosfera "La Sepultura". SEMARNAT, México, D.F.

Terborgh, J. 1977. Bird species diversity on an Andean elevation gradient. Ecology, 58:10071019.

Toledo, V. 1988. Pleistocene changes of vegetation in tropical Mexico. Pp. 93-111, en: Biological Diversification in the Tropics (G.T. Prance, ed.). Columbia University Press, Nueva York., E.U.A.

Vargas-Contreras, J.A. y A. Hernández-Huerta. 2001. Distribución altitudinal de la mastofauna en la Reserva de la Biosfera “El Cielo”, Tamaulipas, México. Acta Zoologica Mexicana (nueva serie), 82:83-109.

Whittaker, R.H. y W.A. Niering. 1975. Vegettion of Santa Catalina Mountains, Arizona: a gradient analysis of the south slope. Ecology, 46:429-452. 

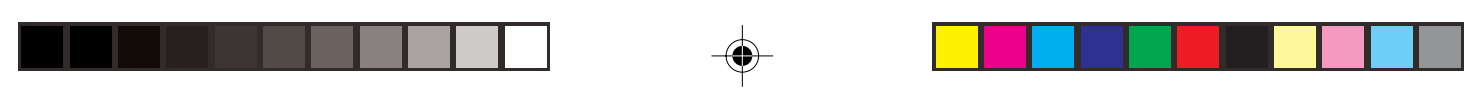

Diciembre 2002 Monteagudo, D. y L. León - Patrones altitudinales...

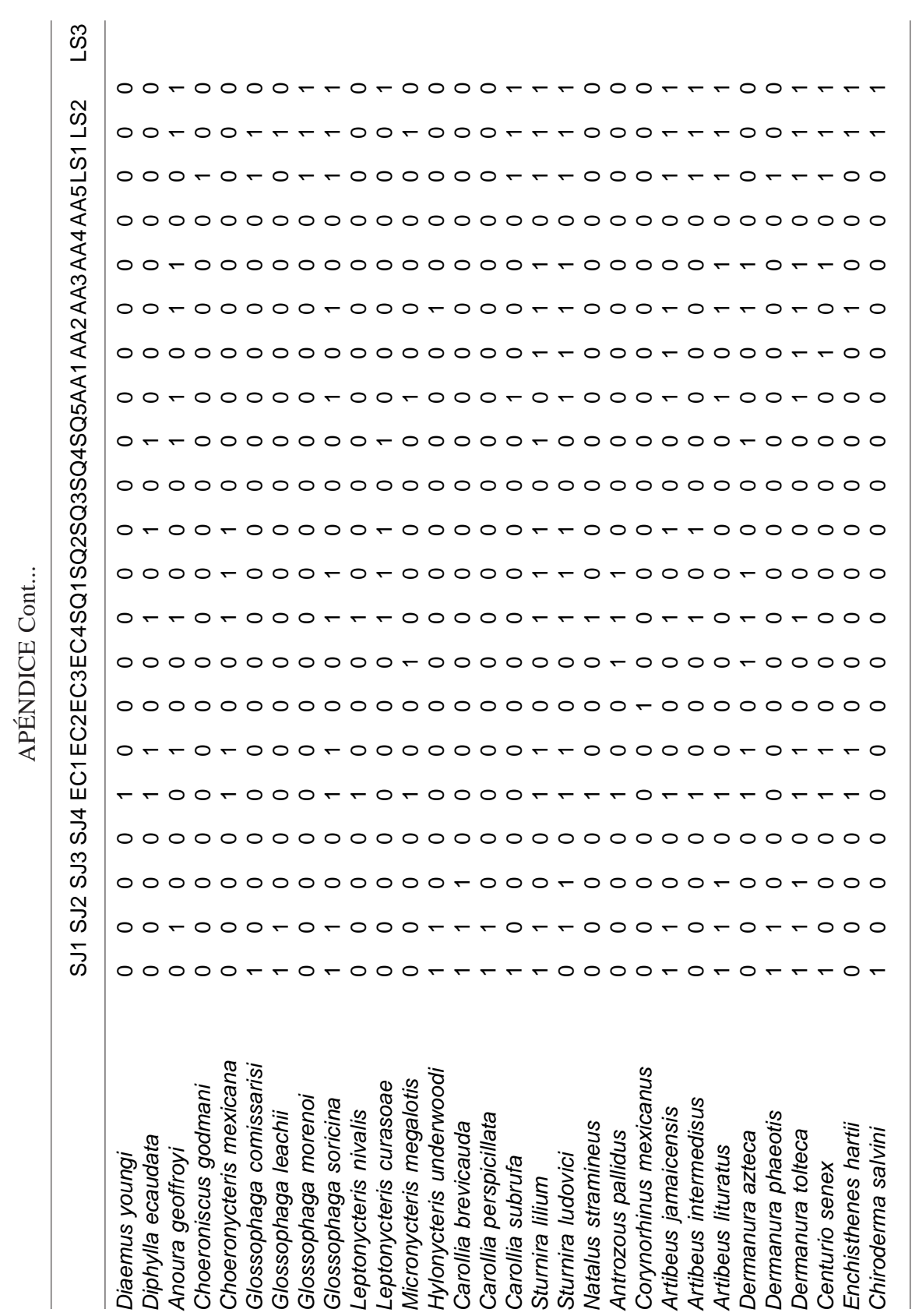



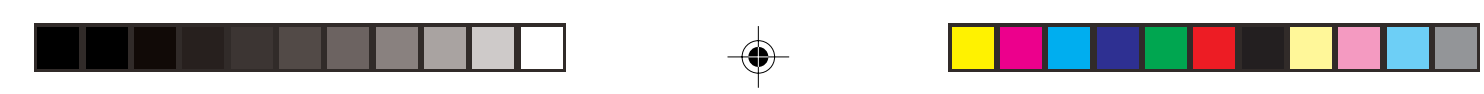

Diciembre 2002 Monteagudo, D. y L. León - Patrones altitudinales...

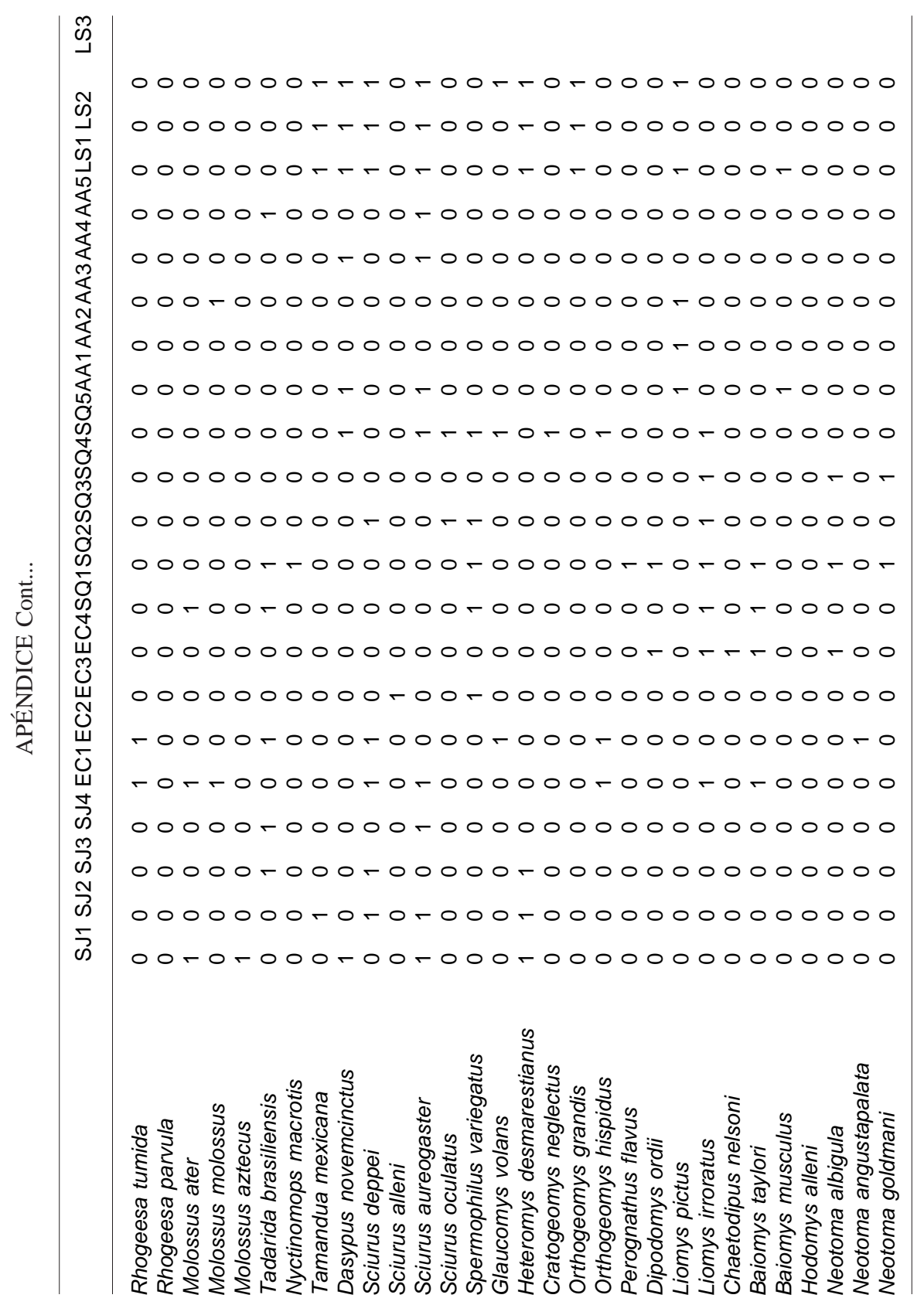



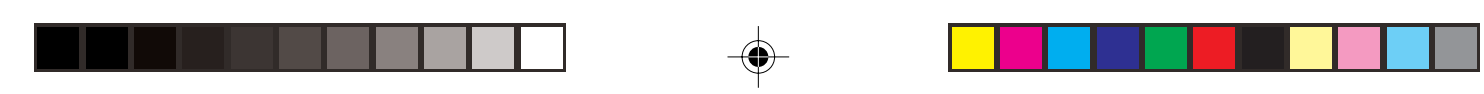

Diciembre 2002 Monteagudo, D. y L. León - Patrones altitudinales...

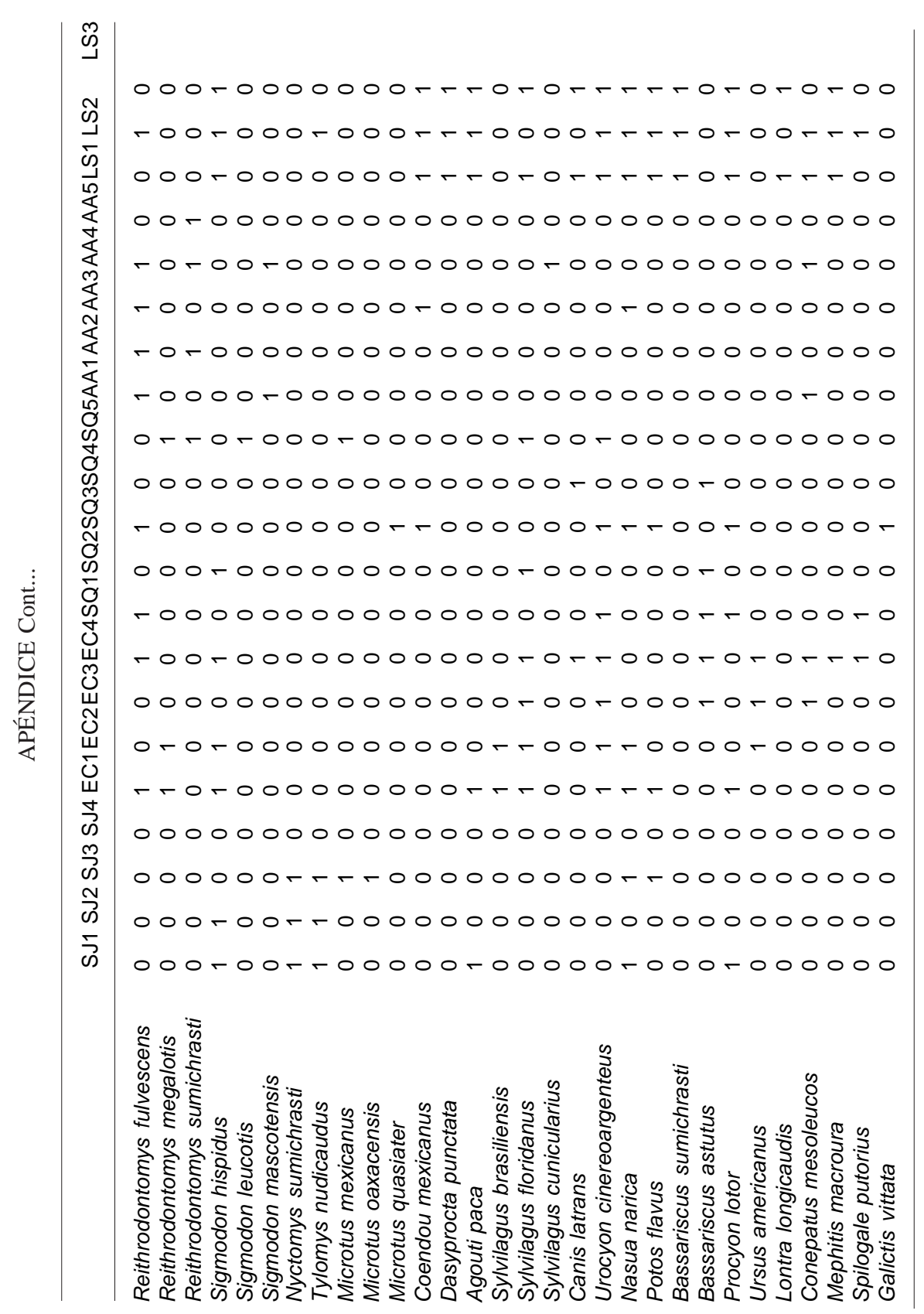


